\title{
Ethical challenges of informed consent in prehospital research
}

\author{
James Thompson, MD
}

\begin{abstract}
Informed consent to participation in research is an important protector of potential subjects' rights and autonomy. Ethical research involving critically ill people is challenging because their medical condition often makes obtaining informed consent impossible. This is especially true in the prehospital setting, where additional barriers to obtaining informed consent exist. A recently published Canadian policy (Tri-Council Policy Statement: Ethical Conduct for Research Involving Humans) specifies circumstances under which an exception to the requirement for informed consent may be granted so that vulnerable individuals are not denied the potential benefits of participating in research. This article reviews the rationale for the Tri-Council Policy Statement and illustrates some problems with its application in the context of a Canadian prehospital study on continuous positive airway pressure. A new risk analysis model and a national research ethics board are discussed as possible ways to facilitate interpretation and application of the current exception of informed consent policy.
\end{abstract}

Key words: prehospital, research, informed consent, exception to informed consent, continuous positive airway pressure

\begin{abstract}
RÉSUMÉ
Le consentement libre et éclairé avant la participation à un projet de recherche est une garantie de protection importante des droits et de l'autonomie des sujets pressentis. La recherche éthique impliquant des personnes gravement malades représente un défi puisque la gravité de leur état empêche souvent ces personnes de donner leur consentement libre et éclairé. Une telle situation prévaut particulièrement dans le milieu pré-hospitalier où il existe d'autres entraves à l'obtention d'un consentement éclairé. Une politique canadienne publiée récemment (Énoncé de politique des Trois Conseils : Éthique de la recherche avec des êtres humains) précise les circonstances selon lesquelles une exception aux exigences de consentement éclairé pourrait être accordée afin que des individus vulnérables ne se voient pas refuser les bienfaits potentiels de la participation à une recherche. Le présent article passe en revue la synthèse de l'Énoncé de politique des Trois Conseils et illustre certains problèmes liés à son application dans le contexte d'une étude pré-hospitalière canadienne sur la ventilation sous pression positive continue. On discute d'un nouveau modèle d'analyse des risques et de la mise en place d'un comité national d'éthique en recherche comme moyens de faciliter l'interprétation et l'application de l'exception actuelle de la politique de consentement libre et éclairé.
\end{abstract}

\section{Introduction}

Voluntary participation in clinical research by human subjects is essential if advancements in the understanding and treatment of disease are to occur. Rational well designed protocols and potential subjects' informed consent prior to their participation are essential to protect subjects from harm when they are involved in studies. For critically ill persons participating in clinical trials, the stakes are especially high: a substantial proportion die, and therapeutic in- 
terventions may offer tremendous (potentially life-saving) benefits. This subset of individuals is uniquely vulnerable because the nature of their illness may render them incapable of providing informed consent. The prehospital environment is rich in opportunities for research involving time-sensitive resuscitative interventions but this environment also presents unique challenges for investigators wishing to ethically enrol critically ill subjects into such studies. Considerable scientific and public debate around this issue has resulted in policies, both in Canada and the US, that allow for an exception to the requirement for informed consent (henceforth referred to as "an exception") in specific emergency health situations. ${ }^{1}$

This article reviews the issues surrounding informed consent in critically ill persons, with a particular focus on the prehospital setting. By describing one research team's experience with the ethics approval process for an ongoing Canadian prehospital study, some of the problems with the application of the current Canadian policy governing an exception will be illustrated. Potential strategies to facilitate the ethical analysis of prehospital critical care research protocols, especially issues surrounding informed consent, are discussed.

\section{Barriers to informed consent in the critically ill}

Informed consent is only valid when given freely by an individual who can communicate and who has decisionmaking capacity (i.e., the ability to understand the risks, benefits and consequences of a particular course of action in the context of a stable set of values). People who are critically ill are vulnerable because their medical condition diminishes decision-making capacity and ability to communicate. It is therefore harder for them to formulate and express autonomous wishes. Though some such people may remain conscious, it is highly unlikely that they could provide a valid informed consent to participation in research. ${ }^{2-6}$ Moreover, health care providers cannot reliably identify those critically ill individuals who retain the capacity to give informed consent without using lengthy, formal capacity-assessment tools. ${ }^{3,6}$ Additionally, when confronted with a request to participate in a research study, individuals in extremis (or their surrogates) may agree to enrol under the belief it will expedite their care. ${ }^{7.8}$

The issues above have profound consequences for those wishing to enrol critically ill individuals into research studies. The life-threatening nature of these individuals' conditions demands urgent resuscitation, precluding the use of lengthy capacity-assessment tools by caregivers, whether physician, nurse or paramedic. The assumption has there- fore been made that no critically ill individual at the time of initial resuscitation has the ability to provide valid informed consent. Other means have been sought to protect such individuals' autonomy and to facilitate their participation in research.

The current standard of practice is to call upon surrogate decision-makers to give medical care providers their best estimate of the treatment wishes of individuals incapacitated by severe illness. However, this is an imperfect approach; when patients being treated in the intensive care unit and their family members were surveyed separately regarding participation in 2 fictional studies, surrogates misrepresented the wishes of their family members up to $20 \%$ of the time. ${ }^{9}$ Such a discrepancy may be significant, particularly if the research holds little potential for direct benefit to the sick individual.

\section{Barriers to informed consent in the prehospital setting}

In addition to incapacitated patients and potentially unreliable surrogate decision-makers, the prehospital setting creates other unique challenges for those wishing to enrol critically ill individuals into research trials. Perhaps the most important issue is that of time. Paramedics are mandated, often by written protocols, to stabilize and transport patients as expeditiously as possible. Timely transport is a universally accepted standard in most circumstances. Attempting to conduct full informed consent discussions onscene may lead to unacceptably long delays in patient transport, and may also adversely affect the quality of any consent obtained..$^{10}$

Searching for a surrogate decision-maker could also unacceptably prolong prehospital scene times. In fact, many critically ill patients are alone at the time of paramedic arrival. In one study surrogate decision-makers were present at only $57 \%$ of out-of-hospital cardiac arrests, and approximately $40 \%$ of surrogates who were present had difficulty understanding an informed consent discussion. ${ }^{11}$

A final issue is that of the therapeutic window: the time period during which administration of an intervention is likely to produce a clinically beneficial effect. In general, the therapeutic window for resuscitative interventions in the prehospital setting is very short. For example, electrical cardioversion of ventricular fibrillation should ideally occur within 4 minutes of cardiac arrest to minimize irreversible brain damage. ${ }^{12}$ Patients suffering acute myocardial infarction derive more benefit from fibrinolytic therapy the earlier it is administered, ideally within 30 minutes from the onset of symptoms. ${ }^{13}$ Delaying critical 
therapeutic interventions to conduct an informed consent discussion in such situations could cause significant harm.

\section{The need for prehospital research in the acutely critically ill}

Paramedics are often the first medical contact with acutely ill individuals and are uniquely placed to provide critical time-sensitive interventions. However, most of the prehospital interventions currently provided have been transferred directly from the hospital setting, without rigorous evaluation of their prehospital effectiveness. ${ }^{14,15}$ This approach has been shown to be problematic. For example, numerous studies have shown that prehospital administration of a fibrinolytic for acute ST-segment elevation myocardial infarction is both feasible and efficacious, but other studies demonstrate that when costs and numbers of eligible patients are included in the analysis, such therapy becomes ineffective. ${ }^{16-18}$ Similarly, although endotracheal intubation is the modality of choice for definitive airway management of pediatric patients in many prehospital systems, Gausche and coworkers ${ }^{19}$ found no benefit in terms of survival or neurological outcome of prehospital endotracheal intubation over bag-valve-mask ventilation in an urban setting. In fact, the goal of the Ontario Prehospital Advanced Life Support study is to define which, if any, prehospital life support interventions improve patient outcomes. ${ }^{20}$

Others have argued the issue from a more theoretical standpoint. Alpert ${ }^{21}$ states "[the patient's] inability to give informed consent and the common absence of an appropriate individual to provide surrogate consent should not be a limiting factor in the development of better interventions." She goes on to argue that it is unethical to exclude the critically ill from the potential individual and societal benefits of participating in research.

Thus, there is both a practical and ethical imperative to carry out prehospital research in critically ill patients. Because potential subjects cannot be afforded the protection of informed consent, regulatory and advisory bodies have sought ways to protect these individuals' basic rights and respect their autonomy, while still allowing this important research.

\section{Exception to informed consent}

The long and, at times, colourful history of policies concerning an exception to the requirement for informed consent for research in emergency health situations has been well documented. ${ }^{1,22}$ A consensus has emerged that in some emergency situations subjects can be enrolled in research trials without their informed consent, provided the trial meets certain stringent requirements. This was first formalized in a $1996^{23}$ combined US Food and Drug Administration (FDA) and Department of Health and Human Services "final rule" (henceforth referred to here as the "Final Rule"). In 1998 the Medical Research Council (now the Canadian Institutes of Health Research), the Natural Sciences and Engineering Research Council of Canada, and the Social Sciences and Humanities Research Council of Canada jointly issued the "Tri-Council Policy Statement: ethical conduct for research involving humans" (TCPS). ${ }^{24}$ Article 2.8 in Section $2(\mathrm{~F})^{25}$ of the TCPS deals specifically with an exception to informed consent in emergency health situations (see Table 1). These councils require that all research funded by them be carried out in accordance with this policy, and thus, research ethics boards (REBs) use the TCPS routinely in their assessment of new protocols. The minimum requirements that must be met for a protocol to qualify for an exception set forth in the 2 policies are similar, although the US "Final Rule" has several additional stipulations: consultation with the community in which the

\section{Table 1. Exception to the requirement for informed consent guidelines in the Tri-Council Policy Statement ${ }^{25}$}

The REB may allow research that involves health emergencies to be carried out without the free and informed consent of the subject or of his or her authorized third party if ALL of the following apply:

a) A serious threat to the prospective subject requires immediate intervention; and

b) Either no standard efficacious care exists or the research offers a real possibility of direct benefit to the subject in comparison with standard care; and

c) Either the risk of harm is not greater than that involved in standard efficacious care, or it is clearly justified by the direct benefits to the subject; and

d) The prospective subject is unconscious or lacks capacity to understand risks, methods and purposes of the research; and

e) Third-party authorization cannot be secured in sufficient time, despite diligent and documented efforts to do so; and

f) No relevant prior directive by the subject is known to exist.

When a previously incapacitated subject regains capacity, or when an authorized third party is found, free and informed consent shall be sought promptly for continuation in the project and for subsequent examinations or tests related to the study.

REB = research ethics board

Reproduced with the permission of the Natural Sciences and Engineering

Research Council of Canada; the Social Sciences and Humanities Research Council of Canada and the Canadian Institutes of Health Research. 
research is to occur; public disclosure of the design, risks and benefits of the trial; an independent data safety and monitoring board; and publication of the results after completion of the trial. ${ }^{23}$ The interpretation and application of the community consultation and public disclosure requirements of the Final Rule has proven to be extremely problematic. ${ }^{26,27}$

The TCPS exception model fits the prehospital acute care setting well, circumventing many of the barriers listed above. A checklist of study inclusion and exclusion criteria (ostensibly meeting the criteria set forth in the TCPS) could replace a lengthy informed consent discussion, requiring little extra documentation by paramedics. Informed consent discussions around continued participation in a trial could subsequently occur in hospital after treatment has been provided, thereby reducing the potential for coercion.

Experience using an exception has been limited, especially in the prehospital setting. Only 2 prehospital studies have been published to date utilizing the Final Rule in the US. ${ }^{28,29}$ Two recent Canadian prehospital cardiac arrest studies $^{30,31}$ received REB approval for waived consent (i.e., patients are enrolled without informed consent and are not approached afterward for informed consent to continue participation in the trial); however, both were prior to publication of the TCPS in 1998. In the ALIVE study ${ }^{30}$ the requirement for consent at the time of enrolment was waived, although some patients were approached for consent once in hospital (Robert Gelaznikas, coauthor of the study: personal communication, Aug 2002). Similarly, Abu-Laban and colleagues were allowed to forego the normal consent procedures in the TPA in PEA Study after they argued "that during a life-threatening event, a reasonable person would consent to potentially life-saving experimental intervention if it were possible to do so." ${ }^{31}$

The Halifax Prehospital Continuous Positive Airway Pressure (CPAP) Study is currently underway with the goal of determining whether the prehospital application of continuous positive airway pressure to patients with acute respiratory failure decreases the need for subsequent endotracheal intubation. At the time of protocol submission, the investigators argued that although patients entering the study would be conscious, many would lack capacity to provide consent, and identification of those with capacity in the field would be impractical. They thus proposed a protocol for study entry utilizing an exception as detailed in the TCPS. After approximately 18 months of negotiation, the investigators and the local REB finally agreed upon an enrolment protocol utilizing an exception (Fig. 1). In addition to the requirements of the TCPS, the REB required that a short "verbal assent" statement be read to all patients prior to their enrolment to give them (or their surrogate if present) the opportunity to refuse participation. An independent data and safety monitoring board is also overseeing the study.

\section{Discussion}

The process of obtaining REB approval of an enrolment protocol utilizing an exception for the Halifax Prehospital CPAP Study raised 2 important issues: the REB requirement for additional patient protections beyond those stipulated by the TCPS, and the unusually lengthy approval process. Because experience in the application of the TCPS to prehospital critical care research is limited, it is difficult to know whether these issues are a local or national phenomenon. Either could pose significant barriers to further prehospital research in Canada, however, and each is addressed below.

By stipulating minimum conditions under which an exception is acceptable, the TCPS and Final Rule provide models for the analysis of the risks and benefits of proposed protocols. However, there is evidence these policies do not reflect societal consensus. A recent study found that only $73 \%$ of a sample of US emergency department patients would enrol in a research trial under an exception. ${ }^{32}$ This dropped to $50 \%$ if the risks involved in the trial were high. ${ }^{32}$ Such lay person discomfort with an exception model may be reflected in the local Halifax REB's ruling that, when available, surrogates have final say regarding enrolment, even if this contravenes patients' stated preferences.

McRae and Weijer ${ }^{33}$ recently argued that an overly literal interpretation of the exception policies may expose vulnerable research subjects to unjustified risk, thus harming the very people the policies were meant to protect. To address this problem, they propose a "risk component analysis," in which the risks posed by participation in a research trial are divided into 2 categories: those associated with the investigational therapeutic intervention and those posed by other nontherapeutic study procedures (e.g., extra blood samples, medical record review). A different ethical test is applied to each category to determine whether an exception can be justified. For investigational therapeutic interventions, they argue that the associated risks are ethically justified if clinical equipoise exists as to optimal treatment for the condition under study (i.e., if there is no consensus in the literature or among experts as to which treatment, standard or investigational, is superior). The risks posed by other nontherapeutic study procedures must be "no more than the risks of daily life" to be ethically justifiable. They argue that studies that meet such criteria, in addition to ful- 
filling the other TCPS or Final Rule requirements, could ethically apply an exception. Whether such an approach would increase public and REB comfort with the use of an exception remains to be seen.

McRae and Weijer's risk component analysis approach does not directly address the issue of enrolling conscious but incapacitated subjects into studies under an exception. Fost $^{34}$ suggests "no reasons are needed to justify saying 'no' to being a research subject." No literature could be found to help guide the Halifax REB in addressing this issue. The board's solution was to require that a verbal assent statement be read to potential subjects and their surrogates (if present) at the time of enrolment. Otherwise, eligible patients who refuse participation cannot be enrolled in the trial. The REB further stipulated that surrogate decision-makers be allowed to veto subjects' enrolment, even if the subjects themselves do not refuse participation. It would appear that in making its decision, the REB felt family members could more accurately determine subjects' autonomous wishes than could the investigators or the critically ill subjects themselves. Clearly, national, societal and scientific consensus on the interpretation and implementation of the TCPS in such situations would aid both investigators and REBs in the design and approval of future studies.

Despite the fact that an acceptable enrolment protocol was eventually negotiated, the time and resources required to obtain an exception for the Halifax CPAP Study were far in excess of the average 5 to 14 hours usually required to obtain REB approval. ${ }^{35}$ Steinbrook ${ }^{36}$ identifies 2 conflict-

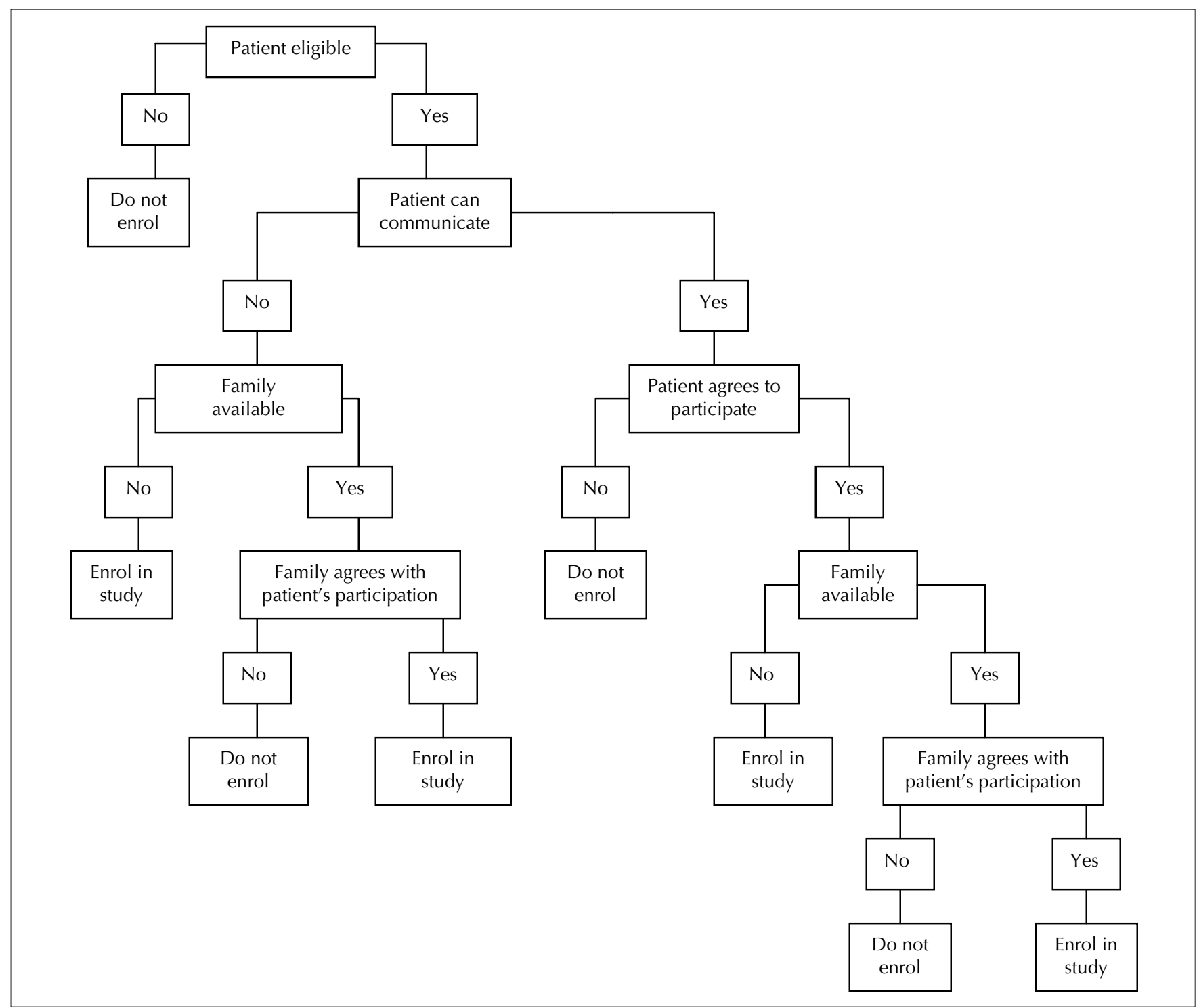

Fig. 1. Algorithm for enrolling patients in CPAP Study 
ing viewpoints regarding the role of the REB: "that increased oversight of clinical research is essential" and "that greater protection of research subjects will interfere with medical progress." Christian and coworkers ${ }^{37}$ argue that "the burdens associated with securing approval from the local IRB for [multicentre cancer treatment] studies limits [physician-investigators'] interest in participation." Centralized REBs have been proposed as one mechanism to facilitate more rapid, independent review of pharmaceutical company sponsored research and complex multicentre trials. ${ }^{37-39}$ Early success with this approach has recently been reported in the context of multicentre studies of cancer treatment. ${ }^{37}$ In this model, the responsibilities and authority of the central and local REBs are formally divided: a central panel of experts with extensive REB experience reviews each protocol and then submits it to the local REB, which may conduct a full review or a more efficient "facilitated review." ${ }^{\prime 37}$ Such an approach might be useful in reviewing ethically complex protocols involving an exception over a reasonable time frame.

\section{Conclusions}

Prehospital research involving critically ill individuals is facilitated by an exception to the usual requirement for informed consent as outlined in the TCPS. As the REB approval process for the Halifax Prehospital CPAP Study demonstrates, however, the practical application of the TCPS can be problematic. A national REB, and an improved risk analysis process, may help local REBs when reviewing such protocols. As Slater states, ${ }^{40}$ however, "regardless of the type of review board (central, local, or contract), it does not have a direct responsibility to the patient. That belongs to the local primary investigator."

Competing interests: Dr. James Thompson is the principal investigator for the Halifax Prehospital CPAP Study.

Acknowledgements: I thank Dr. David Petrie, Ms. Stacy Ackroyd-Stolarz, and Dr. Merril Pauls for their valuable comments on earlier drafts of this manuscript.

\section{References}

1. Gray JD. The problem of consent in emergency medicine research. CJEM 2001;3(3):213-8.

2. Burdick WP, Cassell EJ, Leon AC, Kaufman SG. Preliminary evidence of impaired thinking in sick patients. Ann Intern Med 2001;134:1120-3.

3. Smithline H, Mader TJ, Crenshaw BJ. Do patients with acute medical conditions have the capacity to give informed consent for emergency medicine research? Acad Emerg Med 1999;6:776-80.

4. Prentice ED. Informed consent: the most important predictor. Acad Emerg Med 1999;6:774-5.

5. Fitten LJ, Waite MS. Impact of medical hospitalization on treat- ment: decision-making capacity in the elderly. Arch Intern Med 1990;150:1717-21.

6. Fitten LJ, Lusky R, Hamann C. Assessing treatment decisionmaking capacity in elderly nursing home residents. J Am Geriatr Soc 1990;38:1097-104.

7. Smith HL. Myocardial infarction: case studies of ethics in the consent situation. Soc Sci Med 1974;8:399-404.

8. Silverman HJ. Ethical considerations of ensuring an informed and autonomous consent in research involving critically ill patients. Am J Respir Crit Care Med 1996;154:582-6.

9. Coppolino M, Ackerson L. Do surrogate decision makers provide accurate consent for intensive care research? Chest 2001; 119:603-12.

10. Grim PS, Singer PA, Gramelspacher GP, Feldman T, Childers RW, Siegler M. Informed consent in emergency research: prehospital thrombolytic therapy for acute myocardial infarction. JAMA 1989;262:252-5.

11. Hsieh M, Dailey MW, Callaway CW. Surrogate consent by family members for out-of-hospital cardiac arrest research. Acad Emerg Med 2001;8:851-3.

12. Emergency Cardiac Care Committee, American Heart Association. Standards and guidelines for cardiopulmonary resuscitation (CPR) and emergency cardiac care (ECC). JAMA 1986;255: 2841-57.

13. Effectiveness of intravenous thrombolytic treatment in acute myocardial infarction. Gruppo Italiano per lo Studio della Streptochinasi nell'Infarcto Miocardico (GISSI). Lancet 1986;1:397-402.

14. Pepe PE, Bonnin MJ, Mattox KL. Regulating the scope of EMS. Prehosp Disas Med 1990;5:59-63.

15. Callaham M. Quantifying the scanty science of prehospital emergency care. Ann Emerg Med 1997;30:785-90.

16. Morrison LJ, Verbeek PR, McDonald AC, Sawadsky BV, Cook DJ. Mortality and prehospital thrombolysis for acute myocardial infarction: a meta-analysis. JAMA 2000;283:2686-92.

17. Weaver WD, Cerqueira M, Hallstrom AP, Litwin PE, Martin JS, Kudenchuk PJ, et al. Prehospital-initiated vs hospital-initiated thrombolytic therapy. The Myocardial Infarction Triage and Intervention Trial. JAMA 1993;270:1211-6.

18. Gibler WB, Kereiakes DJ, Dean EN, Martin L, Anderson L, Abbottsmith $\mathrm{CW}$, et al. Prehospital diagnosis and treatment of acute myocardial infarction: a north-south perspective. The Cincinnati Heart Project and the Nashville Prehospital TPA Trial. Am Heart J 1991;121(1 Pt 1):1-11.

19. Gausche M, Lewis RJ, Stratton SJ, Haynes BE, Gunter CS, Goodrich SM, et al. Effect of out-of-hospital pediatric endotracheal intubation on survival and neurological outcome: a controlled clinical trial. JAMA 2000;283:783-90.

20. Stiell IG, Wells GA, Field BJ, Spaite DW, De Maio VJ, Ward $\mathrm{R}$, et al. Improved out-of-hospital cardiac arrest survival through the inexpensive optimization of an existing defibrillation program: OPALS study phase II. Ontario Prehospital Advanced Life Support. JAMA 1999;281:1175-81.

21. Alpert S. Implementing the final rule. Acad Emerg Med 1999; 6:1188-9.

22. Passamani ER, Weisfeldt ML. 31st Bethesda Conference. Emergency Cardiac Care. Task force 3: Special aspects of research conduct in the emergency setting: Waiver of informed consent. J Am Coll Cardiol 2000;35:862-80.

23. Title 21 of the Code of Federal Regulations, Section 50.24 (21CFR50.24). Exception from informed consent requirements for emergency research (61 FR 51528). US Food and Drug Administration Department of Health and Human Services; Oct 2, 1996. Available: www.accessdata.fda.gov/scripts/cdrh/cfdocs/ cfcfr/CFRSearch.cfm?FR=50.24 (database updated 2002 Apr 1; site accessed 2003 Jan 17). 
24. Tri-Council Policy Statement: ethical conduct for research involving humans. Ottawa: Medical Research Council, Natural Sciences and Engineering Research Council of Canada, Social Sciences and Humanities Research Council of Canada; 1998. Available: www.nserc.ca/programs/ethics/english/ethics /policy.htm (updated 2000 Nov 21; accessed 2003 Jan 17).

25. Free and informed consent: Research in emergency health situations. Article 2.8. In: Tri-Council Policy Statement: ethical conduct for research involving humans. Ottawa: Medical Research Council, Natural Sciences and Engineering Research Council of Canada, Social Sciences and Humanities Research Council of Canada; 1998. Available: www.nserc.ca/programs/ethics/english /sec02.htm\#F (accessed 2003 Jan 17).

26. Shah A N, Surgarman J. Protecting research subjects under the waiver of informed consent for emergency research: experiences with efforts to inform the community. Ann Emerg Med 2003; 41:72-8.

27. Schmidt TA. The legacy of the Tuskegee syphilis experiments for emergency exception from informed consent. Ann Emerg Med 2003;41:79-81.

28. Alldredge B, Gelb A, Isaacs S, Corry MD, Allen F, Ulrich SK et al. A comparison of lorazepam, diazepam, and placebo for the treatment of out-of-hospital status epilepticus. N Engl J Med 2001;345:631-7.

29. Ochs M, Davis D, Hoyt D, Bailey D, Marshall L, Rosen P. Paramedic-performed rapid sequence intubation of patients with severe head injuries. Ann Emerg Med 2002;40:159-67.

30. Dorian P, Cass D, Schwartz B, Cooper R, Gelaznikas R, Barr A. Amiodarone as compared with lidocaine for shock-resistant ventricular fibrillation [published erratum appears in N Engl J Med 2002;347:955]. N Engl J Med 2002;346:884-90.

31. Abu-Laban RB, Christenson JM, Innes GD, van Beek CA,
Wanger KP, McKnight RD, et al. Tissue plasminogen activator in cardiac arrest with pulseless electrical activity. N Engl J Med 2002;346:1522-8.

32. Smithline HA, Gerstle ML. Waiver of informed consent: a survey of emergency medicine patients. Am J Emerg Med 1998;16:90-1.

33. McRae A, Weijer C. Lessons from everyday lives: a moral justification for acute care research. Crit Care Med 2002;30:1146-51.

34. Fost N. Can acutely ill patients consent to research? Resolving an ethical dilemma with facts. Acad Emerg Med 1999;6:772-3.

35. Evaluation of NIH implementation of Section 491 of the Public Health Service Act, mandating a program of protection for research subjects. Review draft final report. NIH contract no. N01-OD-2-2109. Bethesda (MD): Office of Extramural Research, National Institutes of Health; May 1998.

36. Steinbrook R. Improving protection for research subjects. N Engl J Med 2002;346:1425-30.

37. Christian MC, Goldberg JL, Killen J, Abrams JS, McCabe MS, Mauer JK, et al. A central institutional review board for multiinstitutional trials. N Engl J Med 2002;346:1405-8.

38. Tully J, Ninis N, Booy R, Viner R. The new system of review by multicentre research ethics committees: prospective study. BMJ 2000;320:1179-82.

39. Ferris L. Industry-sponsored pharmaceutical trials and research ethics boards: Are they cloaked in too much secrecy? CMAJ 2002;166:1279-80.

40. Slater EE. IRB reform. N Engl J Med 2002;346:1402-4.

Correspondence to: Dr. James Thompson, Department of Emergency Medicine, QE II Health Science Centre, Halifax Infirmary Site, 1796 Summer St. Halifax NS B3H 3A7; 902 473-2020, fax 902 473-3617, thompsoj@dal.ca 